\title{
Aerolíneas de bajo costo en México Competencia modal, intermodal e intramodal
}

\section{Ignacio Javier Cruz}

Rodríguez

Facultad de Estudios Superiores Acatlán, UNAM

Iker_izquierdo1@hotmail.com

\section{Resumen}

Mediante diversos métodos se estimó el impacto que tuvo la entrada de aerolíneas de bajo costo (ABC) al mercado de transporte de pasajeros en México; para ello se distingue una tipología de la competencia en modal, intramodal e intermodal. Se encontró que para todos los tipos de competencia el ingreso de las $\mathrm{ABC}$ resultó provechoso para los usuarios de este tipo de servicios.

Palabras clave: economía del transporte, competencia, transporte de pasajeros 


\title{
Low cost airlines in Mexico. Modal, intermodal and intramodal competition
}

\begin{abstract}
By means of diverse methods this paper estimates the entry impacts of Low Cost Airlines (ABC) on passenger transport in Mexico, making a distinction between a typology of modal, intermodal and intramodal competition. Results show that, for every kind of competition, ABC's entry was useful for users of such services.
\end{abstract}

Keywords: transport economics, competition, passenger transport.

\section{Introducción}

La entrada al mercado de las aerolíneas de bajo costo ( $\mathrm{ABC}$ ) generó diversos impactos llamados modales, intermodales e intramodales. Para probar la hipótesis de que la entrada al mercado de las $\mathrm{ABC}$ generó una mayor competencia en cada uno de los mercados mencionados con los consecuentes beneficios para el consumidor de este tipo de servicios, se estudia cada tipo de mercado con una herramienta diferente para cada uno de ellos con la finalidad de cuantificar los impactos del ingreso de las líneas aéreas de bajo costo en el mercado de transporte de pasajeros.

En la primera parte de este trabajo se mencionan los tipos de competencia y las especificidades de cada mercado. Posteriormente, se aborda el caso de la competencia intermodal en la cual se cuantifica un precio generalizado ampliado (PGA) que mide la diferencia entre los gastos totales en los que incurre un usuario al tomar una u otra modalidad de transporte; asimismo, mediante el cálculo y comparativo de este indicador para las modalidades carretera y aérea se mide la diferencia en pesos que resulta menor en las ABC. Más adelante se presenta el caso de la competencia intramodal de las $\mathrm{ABC}$ con las líneas aéreas de precio regular (LPR); de igual forma se calcula una relación marginal de sustitución (RMS) que cuantifica la cantidad de viajes por LPR que los usuarios estuvieron dispuestos a sustituir por viajes en $\mathrm{ABC}$. Después se analiza la competencia modal mediante el cálculo de índices de concentración, los cuales a su vez son correlacionados con el comportamiento que presenta una de las empresas del sector que ha ganado mercado, a diferencia del resto de empresas cuya participación de mercado ha sido relativamente estable. Por último, se presentan las conclusiones. 


\section{Definición de la tipología de competencia}

La entrada al mercado de las aerolíneas de bajo costo (ABC) en México en diciembre de 2005 detonó tres tipos diferentes de competencia: intermodal, intramodal y modal. Cabe aclarar que por competencia se entiende una lucha entre importantes empresas por la preferencia monetaria de los consumidores del servicio. La competencia intermodal es aquella en la cual compiten empresas de diversas modalidades de transporte; se lleva a cabo en rutas que tienen la particularidad de presentar una distancia relativamente grande entre el origen y el destino involucrado; recorrer dicha distancia ofrece una ventaja para la modalidad transportista que pueda desplazarse con mayor rapidez, pues el usuario de servicios de transporte valúa monetariamente su tiempo; indudablemente, la modalidad que le ofrezca menor tiempo de traslado en una ruta será la elegida. Por ello, este tipo de competencia está determinado por la distancia, si se supone que existe competencia entre $\mathrm{ABC}$ y transporte carretero foráneo de pasajeros la ventaja en largas distancias la tienen las $\mathrm{ABC}$.

El segundo tipo de competencia es la intramodal, ésta se lleva a cabo entre empresas de la misma modalidad de transporte; es decir, es la competencia que enfrentan las empresas de transporte carretero, aéreo, ferroviario y marítimo con otras empresas de su mismo giro. Particularmente en el mercado de transporte aéreo existe una diferencia en transporte de pasajeros, que es el precio del servicio y del cual se deriva el tercer tipo de competencia: la modal, que es aquella que se presenta entre las empresas de una misma modalidad de transporte que tienen un mismo atributo, esto es, entre empresas que componen el mercado de bajo costo; cabe mencionar que antes del ingreso al mercado de las $\mathrm{ABC}$, este tipo de competencia sólo existía entre LPR, pero no entre ABC. Para este trabajo la competencia modal de interés es la que se da entre $A B C$.

Tanto la competencia intermodal como la intramodal son las más abordadas internacionalmente, incluso en otras modalidades de transporte distintas a la aérea. Por ejemplo, Ivaldi y Vibes (2005) analizan la competencia intra e intermodal en el transporte ferroviario para los Estados Unidos. En este mismo sentido Román (2002) estudia los impactos intermodales de la introducción de un tren de alta velocidad en España; ambos estudios logran impactos positivos para la competencia. Por su parte, en el mercado de bajo costo de los Estados Unidos se han encontrado efectos positivos para la competencia, Daraban (2008) encuentra que las líneas aéreas existentes en el mercado reaccionan disminuyendo sus precios antes de la entrada de una ABC. 
Debido a lo anterior, se puede esperar que la introducción de las $\mathrm{ABC}$ al mercado mexicano de transporte aéreo de pasajeros generaría una ventaja para estas empresas con respecto a las empresas que ofrecen precios regulares. Dicha ventaja está determinada por los menores precios que ofrecen las $\mathrm{ABC}$ y las líneas de precio regular para las mismas rutas, que en su mayoría son nacionales. Las ABC iniciaron operaciones en diciembre de 2005 con Interjet como pionera, mientras que Volaris ingresó al mercado en marzo de 2006 y Vivaaerobus en noviembre de 2006. Las otras dos líneas aéreas de bajo costo que operan en el mercado mexicano en el periodo de estudio son Mexicanaclick y Aeroméxico Connect, que pertenecen a grupos empresariales que poseen tanto líneas de bajo costo como líneas de precio regular.

Antes del ingreso al mercado de las $\mathrm{ABC}$, operaban empresas con precios regulares, entre las más importantes se encontraban: Aeroméxico, Mexicana, Aviacsa y Aerocalifornia. Con lo que se puede inferir que el ingreso de las ABC incrementó la competencia en este mercado.

\section{Competencia intermodal}

En esta sección se analiza la competencia intermodal generada por la introducción al mercado de las $\mathrm{ABC}$, cuya competencia intermodal está representada por el transporte carretero. Los usuarios de transporte foráneo por carretera (BUS), antes de la entrada de las ABC, sólo tenían la posibilidad de viajar por aire en las líneas tradicionales cuyos precios resultaban mucho mayores, por lo que esta opción resultaba difícil para estos pasajeros, lo que ocasionaba que sólo los de alto ingreso decidieran utilizar transporte aéreo. La introducción de las ABC implicó que los precios para utilizar un transporte aéreo disminuyeran con relación al que presentaban las LPR dando la oportunidad a usuarios de servicios de transporte de pasajeros de trasladarse por esta vía. Se considera que para un mismo origen destino el traslado vía $\mathrm{ABC}$ es sustitutivo de un servicio por BUS. En el análisis de competencia entre bienes sustitutivos es necesario primero definir el mercado en que estos compiten, también llamado mercado relevante. El artículo 12 de la Ley Federal de Competencia Económica (LFCE) menciona los criterios desde los que se puede determinar el mercado relevante para el caso de la competencia intermodal, lo cual se profundiza en este trabajo. La delimitación se expone a continuación.

a) Servicio relevante. Transporte de pasajeros entre diferentes ciudades que presente el mismo origen-destino en dos modalidades de transporte: 
transporte por carretera y transporte aéreo; este último representado por una aerolínea de bajo costo (ABC).

b) Dimensión geográfica. Se forma por distancias por recorrer entre un origen y un destino. En este estudio se consideran cuatro diferentes rutas; el criterio para determinarlas fue el número de vuelos y corridas de pasajeros en los lugares de mayor frecuencia nacional. Todas las rutas tendrían como origen el Distrito Federal (DF) y los destinos serían Cancún, Guadalajara, Monterrey y Tijuana.

c) Sustitutos. Se considera que las posibilidades de sustitución para el usuario —que tiene la posibilidad de realizar su trayecto en cualquiera de ambas opciones partiendo de un origen hacia un destino determinado en el que dos o más modalidades de transporte ofrecen el servicio— son muy altas.

Debido a que la velocidad del vehículo aéreo es mayor que la del transporte terrestre, para el usuario el primero representa una forma de minimizar los costos indirectos asociados a su viaje. Una medida que puede comparar los costos en los que incurre un usuario ante estas dos posibles modalidades es el precio generalizado ampliado (PGA). Este indicador captura no sólo el precio del boleto que debe pagar el usuario, sino que incorpora una valuación en unidades monetarias del tiempo que el cliente pasa en el vehículo durante el trayecto de su viaje y de los gastos extras en que incurre, como puede ser la alimentación, éstos son los costos indirectos. En presencia de la alta velocidad de desplazamiento de las ABC, el tiempo que pasa el pasajero en el vehículo es menor que si se desplaza por la vía terrestre, lo cual influirá en el valor que tome el PGA. De la misma manera, la baja velocidad del transporte terrestre en viajes largos forzará una erogación mayor por parte del consumidor por concepto de gastos alimenticios, situación que puede no sucede si la misma distancia es recorrida a mayor velocidad.

El cálculo de estos costos se realiza mediante el cómputo del precio generalizado (De Rus, 2003)

$$
P G=P_{i}+v t+\theta
$$

Donde $P G$ es el precio generalizado, $P_{i}$ el precio del viaje que el usuario debe pagar, $v t$ es la valoración monetaria del tiempo empleado en dicho viaje, $t$ el tiempo por recorrer, $v$ el valor unitario del tiempo y $\theta$ representa otros factores que son difíciles de cuantificar, como la comodidad y la seguridad de un viaje, lo cual tendrá en adelante un valor de cero. La decisión del consumidor por una 
u otra modalidad de transporte se basa en minimizar el precio generalizado, pues representa menores gastos.

Este precio generalizado no toma en cuenta otros costos que debe ponderar el usuario en el momento de tomar la decisión del modo de transporte por el que viajará; por ello, se propone un precio generalizado ampliado (PGA); éste es una valuación monetaria de todos los gastos en los que incurre un usuario de servicios de transporte por viajar en una ruta determinada y se expresa de la siguiente manera:

$$
P G A=P_{i}+v t+\theta+E X
$$

Donde $E X$ representa gastos extra que el usuario debe realizar durante un viaje $\mathrm{y}$ entre los que se encuentran los gastos de comida ligados al tiempo del viaje; es decir, si el viaje es largo el usuario deberá consumir alimentos cada ocho horas, lo que significa que tendrá que erogar dinero adicionándolo a los gastos totales asociados al viaje. Este gasto puede no ser necesario en los viajes aéreos debido a su rapidez, pero puede resultar indispensable en los viajes terrestres. Otro gasto ligado a un viaje de muchas horas es el de entretenimiento, el cual no es necesario si el viaje se realiza de forma rápida; este gasto hace referencia a la adquisición por parte del usuario de un libro o revista, o alguna otra cosa que distraiga la atención del usuario mientras realiza su viaje; los demás componentes mantienen su significado. El cálculo y la comparación de PGA para las modalidades de transporte que compiten por el mercado relevante ofrecerán una idea sobre el comportamiento de la demanda de los usuarios de servicios de transporte.

Para determinar las rutas a las que se les calculó un PGA se ha tomado como criterio tener la mayor cantidad de pasajeros transportados en ambas modalidades. Para el caso del transporte carretero, el mayor número de corridas tienen como origen las ciudades de Distrito Federal, Guadalajara y Monterrey, de acuerdo con el IMT(2007). En estas tres ciudades se concentra poco más del 24\% de los pasajeros transportados por esta vía en el nivel nacional y casi el $25 \%$ de las corridas. En el cuadro 1 se aprecian los destinos nacionales de viaje por aire de mayor frecuencia reportados por la Secretaría de Comunicaciones y Transportes (SCT). De esta manera se identifica a Cancún, Ciudad de México, Guadalajara y Monterrey como las ciudades de mayor número de pasajeros en destino. 


\section{Cuadro 1 \\ Pasajeros transportados vía aérea a destinos nacionales}

\begin{tabular}{|c|c|c|c|}
\hline & 2008 & 2009 & 2010 \\
\hline Cancún & 3303 & 3128 & 2930 \\
\hline Guadalajara & 1752 & 1663 & 1447 \\
\hline Monterrey & 1287 & 1236 & 867 \\
\hline Tijuana & 901 & 873 & 832 \\
\hline
\end{tabular}

Fuente: elaborado con datos de Secretaría de Comunicación y Transporte

Puede apreciarse que existe una tendencia a la disminución en el número de pasajeros transportados en los cuatro destinos más concurridos del país. Se presume que esta situación se debe a que en los años 2009 y 2010 hubo recesión económica que llevó a disminuir la demanda de este servicio.

Si el PGA puede ser comparado por los pasajeros para ambas modalidades en un mismo origen-destino estarán ante la posibilidad de tomar una mejor decisión con respecto a su viaje y del dinero que desembolsarán para llevarlo a cabo. El cálculo del PGA se efectuó usando datos correspondientes a agosto de 2009, los cuales están en el cuadro 2. El precio de los boletos se tomó de las páginas web de las empresas de transporte carretero señaladas y de las ABC. Para cuantificar $v$ se tomó un valor de un salario mínimo, es decir, 57.46 pesos por hora (según la Comisión Nacional de Salarios Mínimos), lo que da una cantidad de 459.68 en una jornada laboral por cada veinticuatro horas de viaje'.

\section{Cuadro 2}

\begin{tabular}{c|c|c|c|c}
\cline { 2 - 5 } \multicolumn{2}{c}{} & \multicolumn{2}{c}{ Transporte carretero } & \multicolumn{2}{c}{ Aerolínea de bajo costo } \\
\hline Destino & $\begin{array}{c}\text { Tiempo de viaje } \\
\text { en horas }\end{array}$ & $\begin{array}{c}\text { Precio de viaje } \\
\text { en pesos }\end{array}$ & $\begin{array}{c}\text { Tiempo de viaje } \\
\text { en horas }\end{array}$ & $\begin{array}{c}\text { Precio de viaje } \\
\text { en pesos }\end{array}$ \\
\hline Cancún & 25.30 & 1386 & 2.0 & 1649 \\
\hline Guadalajara & 7.30 & 510 & 1.10 & 1328 \\
\hline Monterrey & 13.0 & 735 & 1.25 & 1066 \\
\hline Tijuana & 19.0 & 1681 & 1.45 & 2360 \\
\hline
\end{tabular}

Fuente: Páginas web ADO, Estrella Blanca, Interjet y Volaris

${ }^{1}$ La información de las distancia entre las rutas se tomó de la página electrónica del atlas de México. 
En el cuadro 2 se pueden apreciar los diferenciales de tiempo de viaje intermodales para un mismo origen-destino, los cuales son considerables. La mayor diferencia es la de la ruta Distrito Federal-Cancún, que es de veintitrés horas y media; mientras que la de menor diferencia está en la ruta Distrito Federal-Guadalajara con poco más de seis horas. Los resultados de la estimación del PGA se muestran en la gráfica 1, donde se puede observar que de las cuatro rutas especificadas sólo en Guadalajara el PGA resulta menor para el transporte por la vía terrestre que para las $\mathrm{ABC}$, implicando que para este origen-destino el usuario de transporte terrestre incurra en menores costos. Para las otras tres rutas el PGA de las ABC es menor que el del transporte terrestre; en dichas rutas los costos en los que incurre el usuario por recorrer dichas rutas por la vía aérea son menores. El diferencial monetario de PGA a favor del transporte terrestre es de 474 pesos, en el caso de la ruta DF-Guadalajara. No obstante, para la ruta DF-Cancún el diferencial en pesos es de 1087 en favor de las ABC. Los otros dos diferenciales toman un valor de 341 pesos a favor de las $\mathrm{ABC}$. Se observa que conforme aumenta la distancia de viaje, el PGA correspondiente aumenta en ambas modalidades de transporte aunque de manera más marcada en el transporte terrestre que en las ABC.

\section{Gráfica 1}

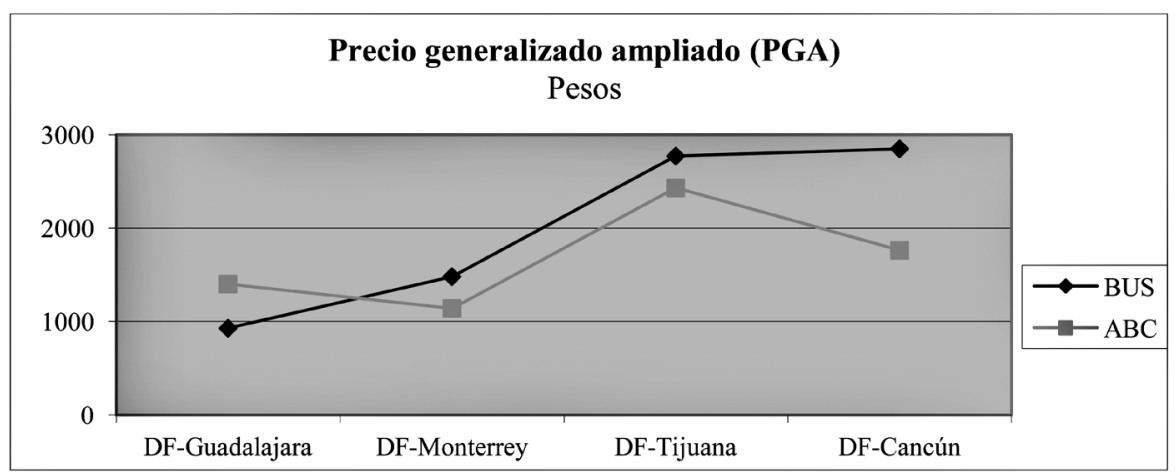

Estos resultados indican que para rutas de viaje que implican distancias largas los usuarios incurrirán en menores gastos si usan una $\mathrm{ABC}$ en lugar del transporte terrestre. Esta valuación indica que si el usuario de servicios de transporte conoce el ahorro de tiempo y dinero de viajar por aire se verá incentivado a dejar de utilizar BUS para sustituirlo por ABC. 


\section{Competencia intramodal}

Para estimar el efecto de la competencia intramodal entre ABC y LPR se estimará una relación marginal de sustitución (RMS), la cual ofrece un cálculo sobre la disposición de un consumidor o grupo de consumidores a sustituir un tipo de servicio por otro. Esta sustitución se presume que se lleva a cabo desde el momento en que los pasajeros que tradicionalmente viajaban por LPR vieron la posibilidad de pagar un precio menor por el mismo tiempo de vuelo entre un mismo origen-destino.

Tradicionalmente para calcular una RMS se utilizan dos más productos o servicios que suelen no estar relacionados; es decir, los sectores involucrados en el calculo de la RMS suelen no competir entre ellos. No obstante, la RMS puede emplearse para dos empresas o sectores que compitan entre sí. En este caso, el usuario de servicios de transporte aéreo pudo sustituir sus viajes en LPR por viajes en ABC; la RMS resultante explicaría qué cantidad de viajes en LPR estuvieron dispuestos a renunciar los pasajeros por viajes en $\mathrm{ABC}$ reflejando competencia entre ellas. Con el teorema de la preferencia revelada, si un consumidor elige una cesta cuando pudo haber elegido otra se dice que prefirió la primera a la segunda; por ello, la idea que se persigue es conocer la disposición de los usuarios por dejar de demandar un vuelo de LPR y sustituirlo por un vuelo de ABC. Como primer paso se debe plantear una función de utilidad. La utilidad es el nivel de satisfacción que obtiene una persona consumiendo un bien o servicio. La utilidad indica una ordenación de preferencias de diferentes cestas de consumo. Por esta razón, el número total de pasajeros con origen-destino nacional transportados será tomado como la variable dependiente, pues los pasajeros por realizar sus viajes debieron obtener cierto nivel de utilidad.

Por su parte, las variables independientes que se han incluido son el número total de vuelos nacionales de las $\mathrm{ABC}$ y el número de vuelos nacionales de las LPR. El periodo para el cual los datos de las variables mencionadas se encuentran disponibles va de enero de 2006 a diciembre de 2009. La estadística descriptiva de los datos que serán utilizados se presenta en el cuadro 3. 


\section{Cuadro 3}

\section{Estadísticos descriptivos}

\begin{tabular}{cccc}
\hline & $\begin{array}{c}\text { Total de pasajeros } \\
\text { nacionales }\end{array}$ & $\begin{array}{c}\text { Vuelos } \\
\text { ABC }\end{array}$ & $\begin{array}{c}\text { Vuelos } \\
\text { LPR }\end{array}$ \\
\hline Media & 941437.94 & 2210.65 & 9755.33 \\
\hline Desv. estándar & 101850.90 & 844.80 & 2687.90 \\
\hline Asimetría & -0.121 & 0.370 & -0.285 \\
\hline Curtosis & 2.361 & 0.865 & 1.405 \\
\hline Mínimo & 884812 & 681 & 4986 \\
\hline Máximo & 1165383 & 3953 & 13469 \\
\hline
\end{tabular}

La función planteada para su estimación es la siguiente:

$$
P=\alpha * A B C^{\beta} * L P R^{\gamma}
$$

Donde $P$ es el número total de pasajeros nacionales transportados, tanto por $\mathrm{ABC}$ como por LPR, que refleja la utilidad obtenida por los usuarios al haber realizado vuelos con origen-destino nacional, $A B C$ es la cantidad de vuelos nacionales realizados por las aerolíneas de bajo costo, $L P R$ es la cantidad de vuelos nacionales que realizaron las líneas de precios regular, $\alpha$ es una constante, $\beta$ y $\gamma$ son coeficientes por estimar que describen las preferencias de los consumidores. Para linealizar la función se aplicaron logaritmos a la misma. Una función parecida a esta especificación se encuentra en Domenich y McFadden (1975). La estimación se realizó mediante mínimos cuadrados ordinarios (MCO) y los resultados se presentan a continuación:

\section{Cuadro 4}

\begin{tabular}{c|c|c}
\hline \multicolumn{3}{|c}{ Resultado de la regresión } \\
\hline & Coeficiente & Error Estándar \\
\hline Constante & $7.0076^{*}$ & 1.056 \\
\hline ABC & $0.2389^{*}$ & 0.054 \\
\hline LPR & $0.5384^{*}$ & 0.073 \\
\hline
\end{tabular}

* Estadísticamente significativo, error estándar entre paréntesis 
Los coeficientes estimados representan las utilidades marginales de los pasajeros por vuelos tanto de ABC como de LPR. En el cuadro 4 se aprecia que los pasajeros obtuvieron en promedio más utilidad por un vuelo en LPR que en ABC. Este resultado es el reflejo de que el número de pasajeros que se transportan por LPR es mayor que los que utilizan $\mathrm{ABC}$ en cada mes de la muestra utilizada. Las pruebas de especificación asociadas a la regresión planteada se presentan en el cuadro 5.

\section{Cuadro 5}

\begin{tabular}{l|c|c|c}
\hline \multicolumn{4}{c}{ Pruebas de especificación } \\
\hline Normalidad & Jarque-Bera & Estadístico & Probabilidad \\
\hline Linealidad & Ramsey-Reset & 1.423238 & 0.662554 \\
\hline Heteroscedasticidad & ARCH & 1.1326 & 0.4200849 \\
\hline Heteroscedasticidad & White & 0.748865 & 0.292899 \\
\hline Autocorrelación & Breusch-Godfrey & 11.97358 & 0.564216 \\
\hline
\end{tabular}

El cálculo de la RMS se muestra a continuación:

$$
R M S=\partial P / \partial A B C / \partial P / \partial L P R=\beta / \gamma=\mathbf{0 . 4 4}
$$

Lo anterior significa que los usuarios estuvieron dispuestos a sustituir casi medio viaje en $\mathrm{ABC}$ por cada viaje en LPR. Este efecto se explica por el diferencial de precios por un mismo servicio en tiempos muy similares reflejándose en una disminución de la demanda de viajes en líneas de precio regular. En la grafica 2 se puede apreciar esta situación, pues el número total de vuelos de las $\mathrm{ABC}$ presenta una tendencia creciente mientras que el número total de vuelos nacionales de las LPR disminuye. Es importante mencionar que este cálculo es general y comprende todas las rutas con origen-destino nacional. Con información más detallada se podría estimar una RMS para cada destino o para diversos segmentos de mercado; sin embargo, esta información no se encuentra desagregada en la base de datos de la SCT. 


\section{Gráfica 2}

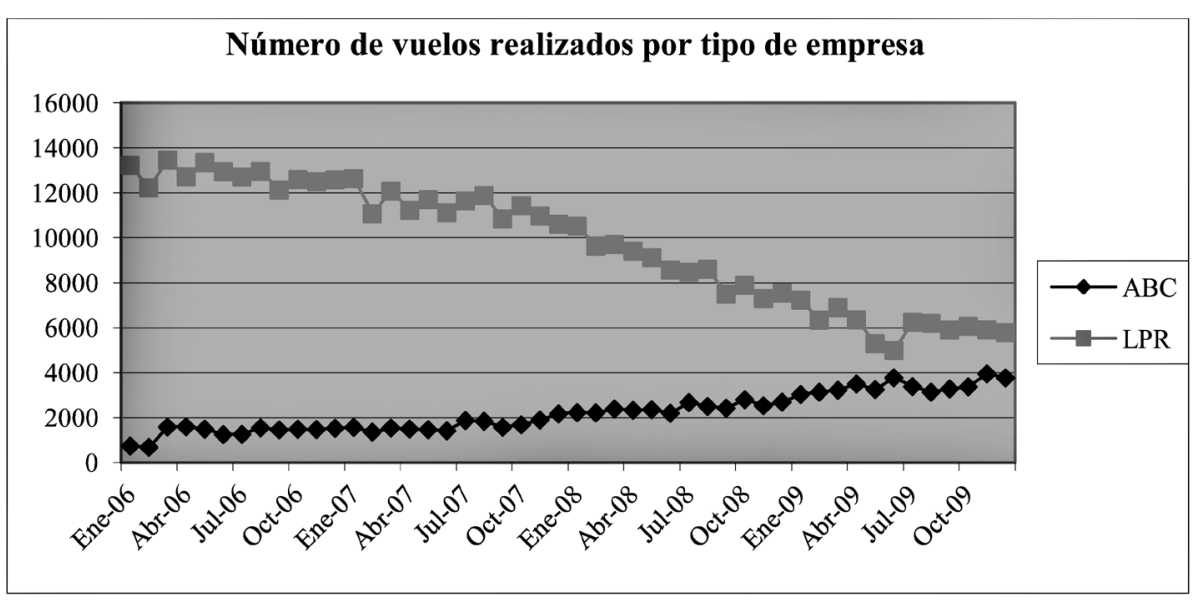

Fuente: elaboración con datos de Secretaría de Comunicación y Transporte

Los resultados y las estadísticas analizadas en esta sección indican que los usuarios han sustituido sus viajes que hacían en LPR por viajes en ABC debido a que los precios que han ofrecido estas últimas son más atractivos para los usuarios incentivando su demanda en detrimento de la demanda de las LPR.

Los bajos precios con los que ingresaron al mercado las $\mathrm{ABC}$ no fueron alcanzados por la LPR aún en sus precios más bajos (discriminación de precios) durante el periodo analizado. Es prudente mencionar que antes de la salida de Mexicana de aviación en agosto de 2010 algunos precios a destinos nacionales de Aeroméxico llegaron a disminuir a niveles un poco más altos que los que ofrecían en promedio las $\mathrm{ABC}$, no obstante, con la salida de Mexicana los precios de las $\mathrm{ABC}$ aumentaron junto con los de las $\mathrm{ABC}$.

\section{Competencia modal}

Para analizar el grado de competencia modal en el mercado de $\mathrm{ABC}$ se han calculado un índice de Hearfindahl-Hirschman (IHH) con información mensual de julio de 2008 a agosto de 2010. Las empresas relevantes para este mercado en el periodo señalado son: Connect, Click, Interjet, Volaris y Vivaaerobus. La fórmula para cuantificar el IHH se presenta a continuación: 


$$
H=\sum_{i=1}^{n} S_{i}^{2}
$$

Es decir, este índice es la suma de los cuadrados de las participaciones de mercado de cada una de las empresas relevantes; en este caso se ha incluido para la construcción del índice el número de pasajeros transportados por cada ABC. El IHH puede tomar valores entre cero y diez mil puntos, si el índice toma el valor del límite superior indica la existencia de un monopolio, mientras que si toma un valor de cero indicaría un mercado plenamente competitivo.

El IHH presenta un promedio de 2236 puntos del periodo junio-diciembre de 2008; mientras que en 2009 el mismo índice presentó un promedio de 2190 y en el periodo de enero-agosto de 2010 el indicador tiene un promedio de 2180 puntos, lo que indica una caída en todo el periodo. La tendencia decreciente del índice se puede ver en la grafica 3 , la cual indica que la competencia en este mercado se ha hecho más fuerte y sugiriere mejoras a favor de los usuarios de estos servicios.

\section{Gráfica 3}

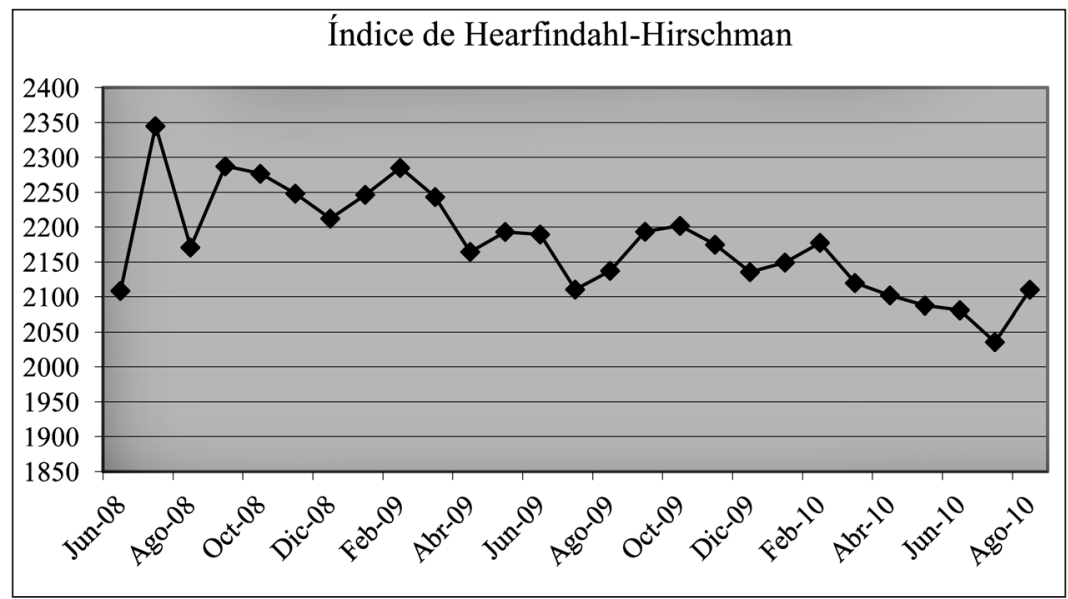

Fuente: elaboración con datos de la Secretaría de Comunicación y Transporte

Cabe mencionar que el mismo índice para el mes de septiembre de 2010 aumentó su valor hasta alcanzar un puntaje de 2753 . Este aumento se debió a la salida del mercado de MexicanaClick que, como puede interpretarse, concentraba el mercado. 
Es de interés resaltar que la empresa que ingresó al mercado más tarde fue Vivaaerobus, pues su ingreso se llevó a cabo hasta noviembre de 2006. Su participación de mercado ha ido aumentando en el periodo de estudio, pues inicia el periodo con un 3.6 por ciento y lo termina con 12.1 por ciento. El resto de la ABC presenta tendencias estables en sus participaciones de mercado como se aprecia en la gráfica 4.

Dada la forma en la que se construye el IHH, se puede asociar su tendencia decreciente con el aumento constante de la participación de mercado de Vivaaerobus. Se ha calculado un coeficiente de correlación para asociar ambas tendencias que resulta en un valor altamente asociativo y negativo de -0.9512 , lo que indica que la disminución en el periodo establecido es lineal e inversamente relacionado con el aumento de la participación de mercado de Vivaaerobus. La tendencia a la disminución que presenta el IHH en la grafica 3 resulta previsible cuando entran al mercado una o más empresas; sin embargo, en esta sección se buscó destacar la fuerte asociación estadística existente entre las variables mencionadas. Por último, resulta evidente que la disminución del IHH representa un indicador positivo para el desempeño de esta industria.

\section{Gráfica 4}

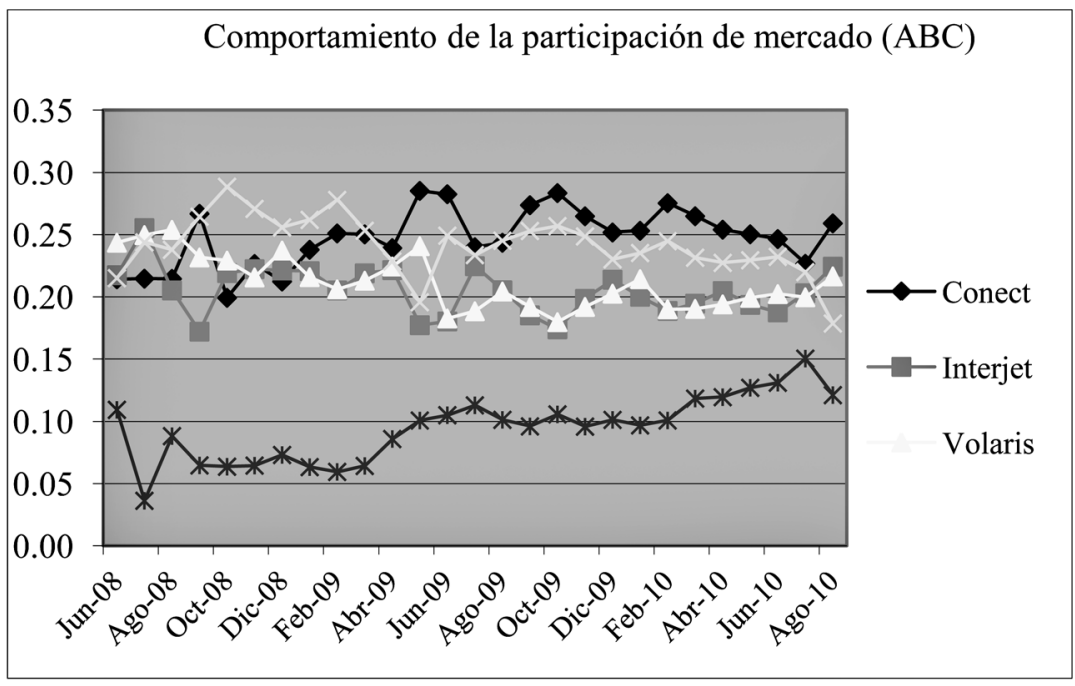

Fuente: Elaborado con datos de Secretaría de Comunicación y Transporte 


\section{Conclusiones}

La incursión en el mercado aéreo de pasajeros de las aerolíneas de bajo costo generó competencia de tres tipos en México: modal, intermodal e intramodal. Se evaluó con diferentes métodos cada tipo de competencia y se encontró impactos positivos para los consumidores derivados de la entrada de este tipo de empresas al mercado. La competencia intermodal se generó con el ingreso de las ABC para ciertos destinos y distancias en las que el usuario obtuvo beneficios al ahorrarse tiempo de traslado. En términos de la competencia intramodal, el consumidor de servicios de transporte obtuvo provecho al tener que pagar un menor precio por recorrer la misma distancia, lo que llevó a que estos consumidores sustituyeran viajes en LPR por viajes en $\mathrm{ABC}$. Por último, el mercado modal en el que compiten solamente aerolíneas de bajo costo muestra, en el periodo analizado, una disminución de su concentración como resultado de la entrada y operación de nuevas empresas que han ganado participación de mercado.

Estos resultados son similares a los encontrados por Behrens y Pels (2009), quienes analizan la competencia intermodal e intramodal entre transporte aéreo y tren de alta velocidad en el mercado de pasajeros en la línea Londres-Paris, donde encontraron que la competencia existe, pero que declinará en el largo plazo. De la misma forma, Turolla, Vassallo y de Oliveira (2008) construyen un indicador para medir la eficacia de reformas tendientes a la liberalización del mercado interestatal de pasajeros en el que la competencia intermodal en líneas aéreas brasileñas benefició a los pasajeros de este tipo de servicios.

\section{Referencias}

Aerolínea de bajo costo Interjet. Disponible en: http: www.interjet.com.mx Aerolínea de bajo costo Volaris. Disponible en: http: www.volaris.com.mx Autobuses de Oriente. Disponible en: http: www.ado.com.mx Autobuses Estrella Blanca. Disponible en: http: www.estrellablanca.com.mx 
Behrens, C. y E. Pels (2009). Intermodal Competition in the London-Paris passenger market: high-speed rail and air transport, Timbergen Institute Discussion Papers.

Chias, L (2003). Transporte y desigualdades territoriales en la región centro. En A. Aguilar, Urbanización, cambio técnico y costo social. El caso de la región centro de México. México: Miguel Ángel Porrua Editor.

Church, J y R. Ware (2000). Industrial Organization: A Strategic Approach. New York: McGraw-Hill.

Daraban, B. (2008). Incumbent responses to low-cost airline entry and exit: A spatial Autoregressive panel data analysis. Research in Transportation Economics 24: 5-24.

De Rus, G. y J. Nombela (2003). Economía del transporte. Barcelona: Antoni Bosch.

Domenich, T. y D. McFadden (1975). Urban Travel Demand. North-Holland Publishing Company.

Gujarati, D. (2003). Econometría. México: McGraw Hill.

Hwa Ryung, L. (2010). Bankruptcy and low cost carrier expansion in the airline Industry, IEW-Cuadernos de trabajo, Universidad de Zurich.

Instituto Mexicano del Transporte 2007 y 2009. Manual estadístico del sector transporte. Disponible en www.imt.mx

Islas, V. (1990). Estructura y desarrollo del sector transporte. México: El Colegio de México.

Ivaldi, M. y C. Vibes (2005). Intermodal and Intramodal Competition in Passenger Rail Transport, CEPR Discussion Paper.

Oum, T. y Y. Zhang (1997). A Note on Scale Economics in Transport. Journal of Transport Economics. 
Ortuzar, J. (1994). Modelos de demanda de transporte. Santiago de Chile: Universidad Católica de Chile.

Román, C. (2002). Competencia intermodal en el corredor Madrid-Zaragoza-Barcelona ante la introducción del tren de alta velocidad. Fundación de estudios de economía aplicada. España.

Secretaría de Comunicaciones y Transportes, "Boletín mensual de estadística operacional. Disponible en http://www.sct.gob.mx/transporte-y-medicina-preventiva/aeronautica-civil/estadisticas/estadistica-aerea-vigente

Turolla, F., M. Vassallo y A. de Oliveira (2008). Intermodal competition in the Brazilian interstate travel market. Revista de Análisis Económico 23 (1): 21-23.

Varian, H. (1999). Microeconomía intermedia. Barcelona: Antoni Bosch. 
